\title{
Controlling the Electron-Beam Interaction with Liquids for In Situ STEM Imaging
}

Patricia Abellan ${ }^{1}$, James E. Evans ${ }^{2}$, Taylor J. Woehl ${ }^{3}$, William D. Ristenpart ${ }^{3,4}$, Ilke Arslan ${ }^{1}$, and Nigel D. Browning ${ }^{1}$

1. Physical Sciences Div., Pacific Northwest National Laboratory, Richland, WA 99352, USA

2. Environmental Molecular Sci. Laboratory, Pacific Northwest National Laboratory, Richland, WA 99352, USA

3. Department of Chemical Engineering and Materials Science, University of California, Davis, CA 95616, USA

4. Department of Food Science and Technology, University of California, Davis, CA 95616, USA

In recent years, there has been a renewed interest in the study of dynamic processes in liquids using environmental stages specifically designed to fit in any transmission electron microscope (TEM) [1]. The possibility of reproducing a similar experimental condition in a liquid environment that can be transferred into different instruments is one of the strengths of hermetically sealed fluid environmental cells. It allows for investigating liquid phase reactions inside different electron microscopes displaying distinct capabilities, such as sub-Angstrom spatial resolution on typical TEM foils- if using an aberration corrected Scanning TEM (STEM) - or high temporal resolution - if using a dynamic TEM (DTEM) [2]. However, in situ studies with liquid stages can be strongly affected by the electron beam and strategies to eliminate or minimize electron beam induced artifacts specific to each experiment are needed [3]. Furthermore, even if the electron dose conditions necessary for reproducing and controlling a reaction are found [3], their application into another instrument operating with different electron optical settings is not straightforward.

When high-energy electrons (typically $80-300 \mathrm{KeV}$ ) irradiate water or an aqueous solution, radiolytic species are formed. Water decomposes upon radiolysis forming the following compounds: $\mathrm{e}_{\mathrm{aq}}^{-}, \mathrm{H}^{\bullet}, \mathrm{O}^{\circ} \mathrm{OH}$, $\mathrm{H}_{2}, \mathrm{H}_{2} \mathrm{O}_{2}, \mathrm{H}^{+}, \mathrm{OH}^{-}$. The chemical species generated may interact with the sample in the fluid cell and trigger undesired reactions. The amount of radiation damage resulting from these beam-induced indirect reactions will depend on the electron dose, incident electron beam energy, and liquid thickness as well as on solution composition and concentration. For instance, in situ growth of nanoparticles in solution induced by the electron beam is typically observed in the (S)TEM [2,3]. Recently, it was demonstrated that at $200 \mathrm{KeV}$ and low beam currents $(\sim 7 \mathrm{pA})$ the in situ growth of $\mathrm{Ag}$ nanoparticles can be predicted by classical theory, and can therefore mimic the synthesis of particles using chemical processes [3]. Even more important, a threshold electron dose rate of 0.5 electrons/Ås ( $<7 \mathrm{pA}$ beam current) below which no nucleation of growth induced by the electron beam occurred was experimentally found [3]. By reproducing such conditions using higher-energy electrons (such as $300 \mathrm{KeV}$ ), radiolysis damage could be reduced, since the cross-section for inelastic scattering - and associated heating effects - would be smaller [4]. In addition, beam broadening would be lowered and thus resolution enhanced, since the cross-section for elastic scatter will be reduced. However, other types of damage, such as knock-on damage could become more pronounced. On the other hand, using smaller acceleration voltages $(80 \mathrm{KeV}, 100 \mathrm{KeV} \ldots)$ could make radiolysis damage much more acute. Figure 1 illustrates the effect of electron beam energy on the in situ growth of silver nanocrystals in solution (see (a) and (c) for $300 \mathrm{KeV}$ and (b) and (d) for $80 \mathrm{KeV}$ ). Note that similar incident beam currents and the same magnification have been used for both experiments. The growth process and resulting nanostructures (size, morphology and crystalline structure) are remarkably different. Here, we use a probe aberration-corrected and 
monochromated Titan electron microscope as a flexible platform to perform a systematic investigation of the effect of different beam settings in aqueous solution in the fluid stage. The beam current is a function of probe size and thus will be controlled by the strength of the $\mathrm{C} 1$ lens - directly determining the spot size - as well as by the gun settings (high tension, extraction voltage, gun lens strength...). See fig. 2(a). Also, the beam current is controlled by the C2 aperture size (See fig 2(b)). Here we will show our approach to allow for scaling those processes to different electron microscope platforms displaying different acceleration voltages [5].

\section{References:}

[1] M.J. Williamson et al, Nature Mater. 2 (2003), p. 532.

[2] J.E. Evans et al, Nano Lett. 11 (2011), p. 2809.

[3] T.J. Woehl et al, ACS Nano 6 (2012), p. 8599.

[4] D.B. Williams and C.B. Carter in "Transmission Electron Microscopy", (Springer, New York)

[5] This work was supported by the National Institutes of Health under NIH grant number RR025032.

Pacific Northwest National Laboratory, is operated by Battelle Memorial Institute for the U.S.

Department of Energy under Contract No. DE-AC05-76RL01830. Development of in-situ stages was supported by DOE NNSA-SSAA grant number DE-FG52-06NA26213, DOE BES grant number DEFG02-03ER46057 and NIH grant number RR025032-01.
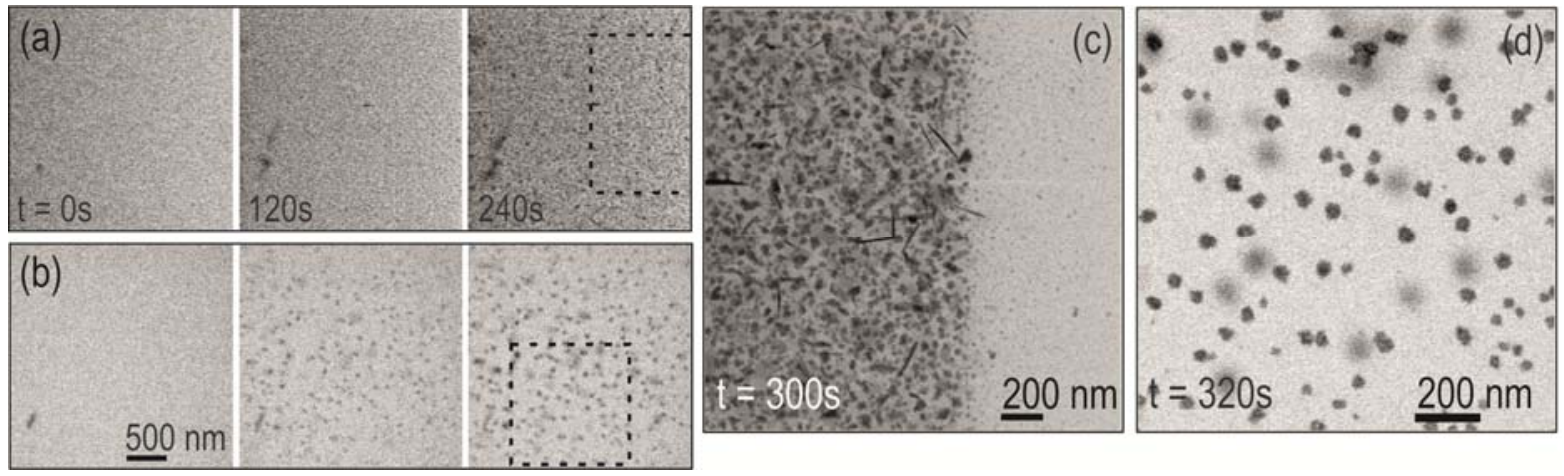

Figure 1. Cropped BF STEM images showing the growth of silver nanoparticles from a $0.1 \mathrm{mM} \mathrm{AgNO}_{3}$ solution for similar beam currents and same magnification $(28500 \mathrm{Kx})$ for times $\mathrm{t}=0,120,240 \mathrm{~s}$. (a) At $300 \mathrm{KeV}$ using $3 \mu \mathrm{s}$ dwell time. (b) At $80 \mathrm{KeV}$ using $6 \mu$ s dwell time. Images (c) and (d) correspond to the dashed square areas in (a) and (b) at the end of the process.
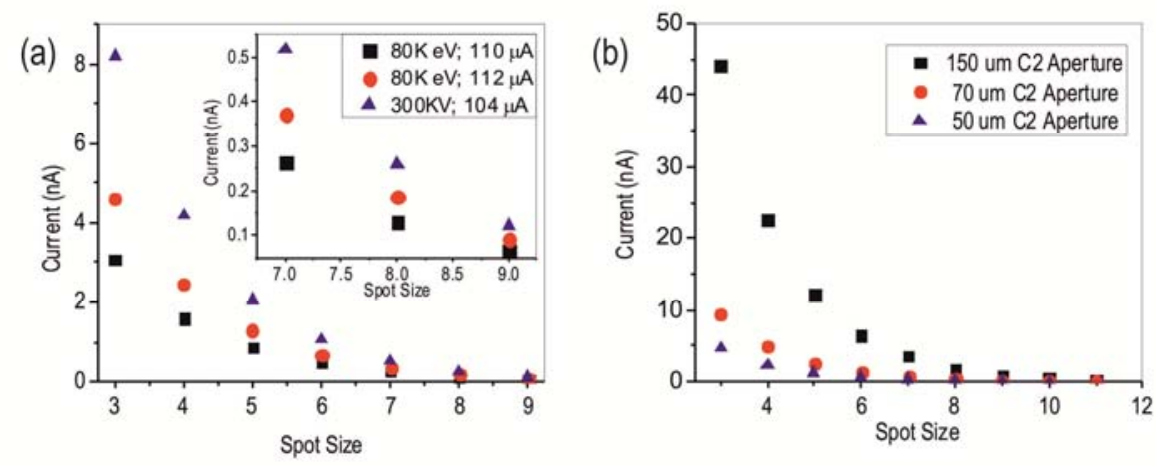

Figure 2. Beam current measured in the screen exposure meter as a function of $\mathrm{C} 1$ strength - spot sizefor different (a) gun settings and (b) C2 aperture sizes. 